CASE REPORT

\title{
Rocking bed and prolonged independence from nocturnal non-invasive ventilation in neurogenic respiratory failure associated with limb weakness
}

\author{
L J Cormican, S Higgins, A C Davidson, R Howard, A J Williams
}

Postgrad Med J 2004;80:360-362. doi: 10.1136/pgmj.2003.010520

\begin{abstract}
A 40 year old mother of three with autosomal dominant scapuloperoneal muscular dystrophy presented with severe neurogenic respiratory failure requiring nocturnal noninvasive ventilation (NIV). Because of the development of profound proximal muscular weakness as a consequence of the progressive nature of her neurological disease, she eventually was unable to apply and remove the facial interface to set up her NIV circuit. She therefore became dependent on her children and carers to start and stop NIV during the night. A rocking bed was successfully employed as an alternative to nocturnal NIV. Ventilation was facilitated by the passive movement of the diaphragm as a consequence of the movement of the abdominal contents under the effect of gravity. Benefit was demonstrated objectively by pulse oximetry and subjectively by the improvement in the patient's symptomatology and continued independence at night. The ease of use of a rocking bed should be borne in mind when the necessity for nocturnal ventilatory support in neuromuscular disease results in the potential loss of independence for a patient.
\end{abstract}

\section{A} 40 year old white mother of three presented with a five year history of increasing shortness of breath on exertion, proximal limb weakness, and orthopnoea. She was unable to carry her youngest child, walk on the steps of a stairs, or lie in a bath. She developed morning headaches, broken and unrefreshing sleep. On examination, deltoid wasting was apparent but there were no fasciculations. Her cranial nerves were intact. She had proximal muscle weakness of her upper and lower limbs, associated with loss of muscle bulk, and reduced tone. Reflexes were present and symmetrical. Her plantar reflexes were flexor and her gait was normal. Abdominal paradox was present and she used her accessory muscles of respiration. Lying and standing vital capacities were 0.46 and 1.14 litres.

She gave a family history of the deaths of her father, paternal aunt, and uncle from respiratory failure in association with peripheral muscle weakness. A formal diagnosis was not known.

Measurement of daytime arterial blood gases while spontaneously ventilating on room air revealed chronic alveolar hypoventilation $(\mathrm{pH} 7.36$, oxygen tension $9.76 \mathrm{kPa}$, carbon dioxide tension $8.79 \mathrm{kPa}$, standard bicarbonate $36.9 \mathrm{mmol} / \mathrm{l}$, base excess +8.9 ).

Domiciliary oximetry (fig l) confirmed significant nocturnal hypoxia with associated heart rate variability (mean oxyhaemoglobin saturation $\left(\mathrm{Spo}_{2}\right) 61.8 \%$, minimum $\mathrm{SpO}_{2}$ $25.2 \%$, oxygen desaturation index (4\%) 25.2 , and 80 6-beats per minute pulse rises per hour).
Her diagnosis was of probable autosomal dominant scapuloperoneal muscular dystrophy with secondary respiratory failure. Unfortunately she refused a muscle biopsy and genetic studies.

She was successfully established on overnight bi-level ventilation (NIPPY II, B\&D Electromedical, Stratford-uponAvon) administered through a nasal interface without supplemental oxygen (inspiratory and expiratory pressures 16 and $3 \mathrm{~cm} \mathrm{H}_{2} \mathrm{O}$, inspiratory: expiratory times 1:3.2 seconds). This was confirmed by improvement in her symptomatology and a repeat nocturnal domiciliary oximetry study.

However over the next eight months she complained of increasing proximal muscle weakness of her upper limbs to the point where she was unable to set up her non-invasive ventilation (NIV) circuit and especially to apply and remove her interface at night. To enable the patient to continue with her beneficial therapy at home additional supportive care would have been required throughout the night.

A rocking bed (fig 2), also known as a body ventilator, was employed as an alternative to her pre-existing bi-level NIV device. This bed, in which the patient slept for the night, rocked her through an arc of amplitude + to $-30^{\circ} 12$ times per minute. This no longer necessitated the use of a nasal mask, nor the help of carers to establish her on NIV, as she merely had to activate or deactivate the rocking bed when required with the use of a switch. The use of alternative modes of ventilation such as negative pressure devices (iron lung, cuirass shell, and body jacket) was considered as they would have not required the use of a facial interface. However, to be established on such modes of ventilation the patient would still have required the help of a carer on a nightly basis. Therefore they offered no advantage in terms of prolonged independence for the patient.

The rocking bed was equally as effective as non-invasive positive pressure ventilation in the treatment of her nocturnal hypoventilation in terms of similar symptomatic benefit and as confirmed by overnight oximetry. Mean $\mathrm{SpO}_{2}$ on room air was $95 \%$ with a minimum of $81 \%$, oxygen desaturation index was 3.66/hour, and heart rate variability was reduced with 16.35 6-beats per minute rises per hour in her pulse rate.

The patient was easily able to manage her alternative mode of ventilation, despite her progressive myopathic disease process, enabling her to prolong her period of independence and improve her quality of life.

\section{DISCUSSION}

The first observation that patients with diaphragmatic insufficiency could be assisted by rocking head up and head down was made by Eve who used the method to resuscitate a

Abbreviations: $\mathrm{NIV}$, non-invasive ventilation; $\mathrm{SpO}_{2}$, oxyhaemoglobin saturation 


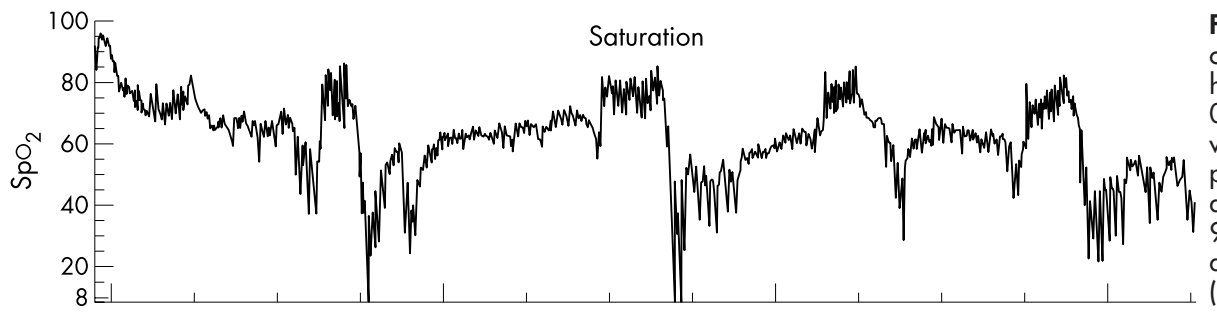

Figure 1 Graphic representation of oxyhaemoglobin $\left(\mathrm{SpO}_{2}\right)$ saturation and heart rate over the time period 0:00 to 06:00 during spontaneous self ventilation on room air. Note the progressive desaturation during assumed sleep from a baseline level of $95 \%$ to a nadir of $25.2 \%$ and the associated heart rate variability (80 6-beats per minute (bpm) pulse

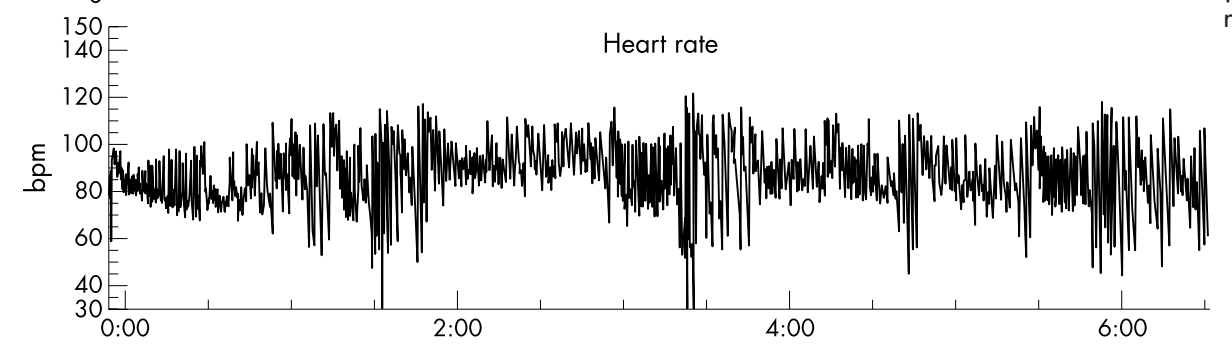
rises per hour).

child dying of diphtheric diaphragmatic paralysis. ${ }^{1}$ Rocking head up and then head down on a regular and frequent basis facilitates inspiration and expiration by the passive movement of the abdominal contents and hence the diaphragm. The navy employed rocking boards during the second world war as a means of resuscitation after drowning. The rocking bed has been used since the 1950s as a single therapeutic modality for respiratory insufficiency in a small number of patients who suffered from neuromuscular disease processes associated with diaphragm weakness and who required NIV. ${ }^{2}{ }^{3}$ However the frequency of use of rocking beds has diminished with time, ${ }^{3}$ to the point where they are no longer available commercially except through specialist home mechanical ventilation units.

The technique has also been employed in the treatment of ventilatory failure caused by diaphragmatic dysfunction due to phrenic nerve injury after cardiac surgery. ${ }^{4}$

In a group of 20 patients with paralytic polio, the percentage increase in tidal volume generated was proportional to the arc through which the bed is rocked, increasing by $45 \%$ for an arc of $32^{\circ}$ and by $66 \%$ for an arc of $40^{\circ} .5$

In a short term comparative study of ventilation by a rocking bed with negative pressure ventilation using a cuirass shell, both methods produced similar increments in $\mathrm{Spo}_{2} .{ }^{6}$

Rocking beds have a valuable role in the management of patients who require NIV because of respiratory failure of neuromuscular origin involving the diaphragm. They also allow freedom from continuous NIV where its use is associated with local complications such as facial and nasal

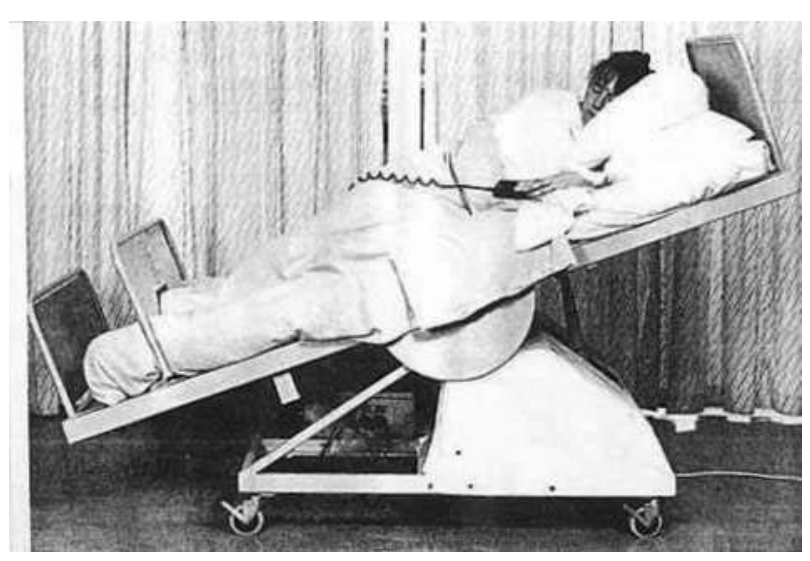

Figure 2 An example of a rocking bed in the head upright position.

\section{Learning points}

- Nocturnal non-invasive respiratory support is frequently required in respiratory failure due to neuromuscular disorders.

- Progressive neuromuscular disorders such as scapuloperoneal muscular dystrophy because of the eventual loss of proximal (and/or distal upper limb) function may result in increasing dependence on carers to establish and discontinue the patient on non-invasive ventilation (NIV).

- A rocking bed does not require the use of a facial or nasal interface, can be activated and deactivated by the patient, and facilitates prolongation of independence from NIV when clinically indicated in these patients.

- When tolerated, it is as effective subjectively and objectively as NIV.

bridge ulceration. Rocking beds are also a useful alternative for patients when NIV is simply intolerable, thus having a palliative role.

For patients with neuorological disease which manifests as respiratory failure and associated limb weakness, the necessity for NIV may accelerate the patient's loss of independence because of the upper limb strength and coordination required for its establishment. A rocking bed, although an antiquated device, can deliver safe and effective ventilatory support for these patients and enable their continued independence.

The use of this device should be borne in mind in the treatment of ventilatory failure in this patient population.

\section{Authors' affiliations}

L J Cormican, Department of Respiratory Medicine, King's College Hospital, London

S Higgins, A C Davidson, A J Williams, Lane-Fox Unit, St Thomas' Hospital, London

R Howard, National Institute of Neurology, London

Correspondence to: Dr Liam J Cormican, Department of Respiratory Medicine, King's College Hospital, Bessemer Road, Denmark Hill, London, SE5 9RS, UK; cormitron@yahoo.com 


\section{REFERENCES}

1 Eve F. Activation of the inert diaphragm by a gravity method. Lancet 1932;ii:995-7.

2 Chalmers R, Howard R, Wiles $C$, et al. Use of the rocking bed in the treatment of neurogenic respiratory insufficiency. Q J Med 1994;87:423-9.

3 St J Trend P, Wiles C, Spencer G, et al. Acid maltase deficiency in adults: diagnosis and management in 5 cases. Brain 1985;108:845-60.
$4 \operatorname{Abd} \mathbf{A}$, Braun N, Baskin $M$, et al. Diaphragmatic dysfunction after open heart surgery: treatment with a rocking bed. Ann Intern Med 1989;111:881-6. 5 Plum $F$, Whedon $G$. The rapid rocking bed: its effect on the ventilation of poliomyelitis patients with respiratory paralysis. N Engl J Med 1951;245:235-41.

6 Goldstein R, Molotiu N, Skrastins R, et al. Assisting ventilation in respiratory failure by negative pressure ventilation and by rocking bed. Chest 1987;92:470-4.

IMAGES IN MEDICINE.

Endoscopic view of a gall stone impacted in the duodenum

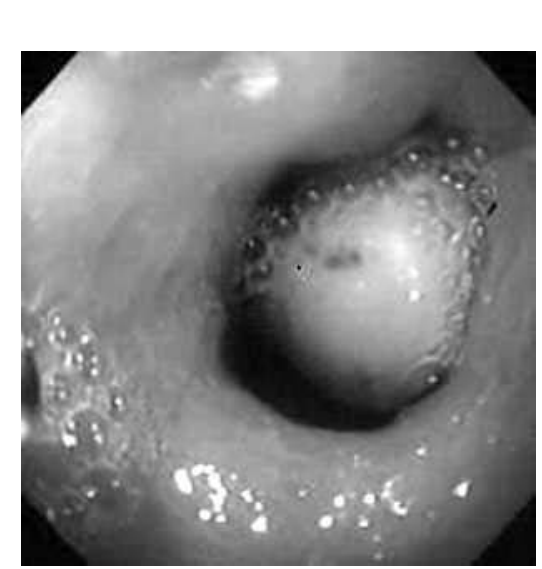

72 year old man presented with severe episodic upper abdominal pain which settled spontaneously. He was left with recurrent vomiting requiring placement of a nasogastric tube. Upper gastrointestinal endoscopy revealed a gall stone impacted in the first part of the duodenum. Endoscopic removal was not possible. At laparotomy, the gall bladder was found to be adherent to the duodenum. The stone was manipulated into the stomach and removed via a gastrotomy. The postoperative period was complicated by an ischaemic cardiovascular accident from which the patient is now recovering.

S Ward-Booth Leicester General Hospital, UK; sally-stephen@wardbooth99.fsnet.co.uk

Figure 1 Endoscopic view of impacted gall stone. 